\title{
Growth factor expression in ophthalmic pterygia and normal conjunctiva
}

\author{
EFSTATHIOS T. DETORAKIS ${ }^{1}$, APOSTOLOS ZARAVINOS ${ }^{2}$ and DEMETRIOS A. SPANDIDOS ${ }^{2}$ \\ ${ }^{1}$ Department of Ophthalmology, University Hospital of Heraklion; ${ }^{2}$ Laboratory of Virology, \\ Medical School, University of Crete, Heraklion, Crete, Greece
}

Received January 20, 2010; Accepted February 19, 2010

DOI: 10.3892/ijmm_00000371

\begin{abstract}
The expression of growth factors in ophthalmic pterygium and phenotypically normal conjunctiva was examined and correlated with the clinical findings. Fifteen specimens of ophthalmic pterygia and 8 specimens of phenotypically normal conjunctiva were examined. Total RNA was extracted from all specimens and mRNA levels for transforming growth factor (TGFB1), vascular endothelial growth factor (VEGFA), basic fibroblast growth factor (FGF2), epidermal growth factor (EGF) and insulin-like growth factor (IGF1) were measured using real-time reversetranscription PCR (qRT-PCR). Differences in the expression of these factors between pterygium and conjunctival specimens were examined, as well as correlations between RNA levels and clinical parameters. mRNA levels for VEGFA and FGF2 were significantly higher in pterygium, compared with conjunctival specimens, whereas the respective differences for other factors examined were statistically not significant. The mRNA levels for VEGFA and FGF2 were also significantly higher in recurrent compared with primary pterygium. Correlations between the mRNA levels and other clinical parameters were statistically not significant for the growth factors examined. The higher levels of FGF2 or VEGFA mRNA in pterygium imply that these factors may be involved in the pathogenesis or clinical behavior of the pterygium, including postoperative recurrence. VEGFA expression may have potential for use in the selective treatment of pterygium with anti-VEGFA agents.
\end{abstract}

\section{Introduction}

Ophthalmic pterygium is a fibrovascular condition of the ocular surface, affecting both conjunctiva and cornea (1).

Correspondence to: Dr Efstathios T. Detorakis, Department of Ophthalmology, University Hospital of Heraklion, 71110 Heraklion, Crete, Greece

E-mail: detorakis@hotmail.com

Key words: pterygium, growth factors, qRT-PCR
Pterygium is common in patients with advanced age and in those exposed to environmental factors, such as sunshine (2). Although benign in nature it can progressively grow in size and threaten vision by occupying the central cornea (3). Surgical excision is often followed by aggressive recurrences (3). Recurrent pterygium is more common in younger patients and is sometimes associated with a family history of pterygium whereas its treatment often requires sophisticated surgery (4). Various theories on the pathogenesis of pterygium have been proposed such as chronic ocular surface inflammation and trauma from exposure to environmental factors, viral infection or a benign neoplastic transformation (5). Several studies have evaluated the potential participation of individual growth factors in pterygium formation and subsequent behavior by employing various techniques, including immunohistochemistry or ELISA (6). This study employed qRT-PCR to assess the expression of a panel of growth factors in pterygia and phenotypically normal conjunctiva, and aimed to associate findings with clinical information. Results may help in further understanding the nature, pathogenesis and clinical course of pterygium and may have clinical implications, taking into account the commercial availability of medications directed against specific growth factors.

\section{Patients and methods}

This is a prospective non-randomized case series. Patients consecutively operated on for primary or recurrent pterygium at the Department of Ophthalmology of the University Hospital of Heraklion in Crete, Greece, were included. Conjunctival samples from patients undergoing cataract surgery who did not have pterygium or a history of pterygium excision were also collected with written permission. Patients with a history of conjunctival surgery (apart from previous pterygium excision), trauma or burn or other ocular surface conditions, including inflammatory and cicatricial conditions of the conjunctiva were excluded to rule out potential effects of these conditions on the expression of the growth factors studied. For the same reason, glaucomatous patients under chronic treatment with topical medications were also excluded. Information recorded for each patient included age, place of current residence, previous pterygium surgery and number of postoperative recurrences, the reported duration of 
pterygium presence of as well as family history of pterygium. Furthermore, the size of pterygium was recorded $(1,2$ or $3 \mathrm{~mm}$ on the corneal surface).

Patients were operated on by the same surgeon (ETD) under local anaesthesia using the same technique (bare sclera excision with adjunctive use of mitomycin $\mathrm{C} 0.2 \%$ for $2 \mathrm{~min}$ ). Following the ink marking of the limbal border, the pterygium head (portion of pterygium covering the cornea) was detached from the anterior corneal surface, and pterygial tissue was dissected at a subconjunctival plane and removed together with the pterygium head. Therefore, tissue samples analyzed included the head of each pterygium as well as pterygial tissue excised sub-conjunctivally along the pterygium body. All pterygia examined were located at the nasal limbal area. Phenotypically normal conjunctival samples were also collected from the same area, to match levels of exposure to environmental factors with specimens of pterygium. Specimens were stored at $-80^{\circ} \mathrm{C}$ until molecular genetic analysis.

Overall 15 pterygial specimens (8 males, 53.33\%) and 8 control specimens (5 males, $62.5 \%$ ) were examined. The age in the pterygium group (mean $\pm \mathrm{SD}$, range) was $64.15 \pm 14.30(38-76)$ years and in the control group $69.32 \pm 17.11(51-80)$ years. In 6 cases $(40 \%)$ pterygium did not extend beyond $1 \mathrm{~mm}$ on the corneal surface, in 5 cases $(33.33 \%)$ it was moderately advanced $(2-3 \mathrm{~mm})$ and in 4 cases $(26.66 \%)$ it was advanced (>3 mm) (7). Patients with pterygium were operated for the first time in 8 cases $(66.66 \%)$, for the second time in $4(26.66 \%)$ and for the third time in 3 cases $(33.33 \%)$. The mean altitude of residence in the pterygium group was $216.73 \pm 19.14(0-800) \mathrm{m}$ and in the control group $192.303 \pm 23.22(0-700) \mathrm{m}$. Pterygium was reported to exist for $9.37 \pm 1.44$ (1-26) years prior to surgical removal. Differences in the altitude of residence and age between the pterygium and control groups were statistically not significant (individual samples t-test), nor was the difference in gender distribution between the pterygium and control groups $\left(\chi^{2}\right.$ test).

A panel of 5 growth factors was studied, including transforming growth factor (TGFB1), vascular endothelial growth factor (VEGFA), basic fibroblast growth factor (FGF2), epidermal growth factor (EGF) and insulin-like growth factor (IGF1). Total RNA was extracted from each specimen using a power homogenizer and TRIzol ${ }^{\circledR}$ reagent (Invitrogen, Carlsband, CA) according to the manufacturer's instructions. Total RNA $(1 \mu \mathrm{g})$ was used in order to synthesize cDNA, using the StrataScript reverse transcriptase kit (Stratagene, La Jolla, CA) as previously described (8). Peptide growth factor mRNA expression was measured using real-time polymerase chain reaction (qRT-PCR) with SYBR-Green I. Primers were designed to span introns, as previously described (9). The gene RPL13A was used as the internal control in order to normalize VEGFA, TGFB1, FGF2, EGF and IGF expression levels. Specifically, $1 \mu 1 \mathrm{cDNA}$ from each sample was amplified in a PCR reaction containing $2 \mathrm{X}$ Brilliant SYBR-Green I QPCR Master Mix, $300 \mathrm{nM}$ of each primer and $30 \mu \mathrm{M}$ ROX passive reference dye, in a final volume of $20 \mu \mathrm{l}$. After an initial denaturation at $95^{\circ} \mathrm{C}$ for $10 \mathrm{~min}$, the samples were subjected to 40 cycles of amplification comprised of denaturation at $95^{\circ} \mathrm{C}$ for $30 \mathrm{sec}$, annealing at $60^{\circ} \mathrm{C}$ for $30 \mathrm{sec}$ and elongation at $72^{\circ} \mathrm{C}$ for $30 \mathrm{sec}$, followed by a melt curve analysis in which the temperature was increased

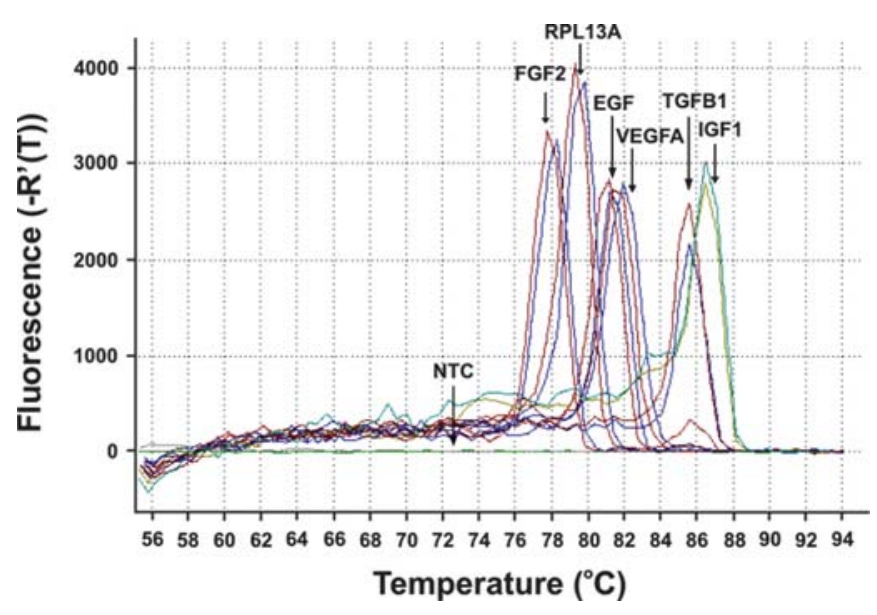

Figure 1. Dissociation curves of the growth factors VEGFA, TGFB1, FGF2, EGF, IGF1 and the normalizer gene RPL13A. Single peaks of representative samples prove the specificity of the amplification products as well as the absence of any by-product or primer-dimer formation. Temperature (Tm) of each amplification product is indicated (NTC, non-template control).

from 55 to $95^{\circ} \mathrm{C}$ at a linear rate of $0.2^{\circ} \mathrm{C} / \mathrm{sec}$. Data collection was performed during annealing and extension with 2 measurements at each step, and at all time points during the melt curve analysis. All PCR experiments were conducted on the Mx3000P RT-PCR thermal cycler using software version 2.0 (Stratagene). Reactions were run in triplicate and peptide growth factor transcript levels were calculated and normalized to each specimen's housekeeping gene mRNA (RPL13A), as well as to the appropriate calibrators, using the $\Delta \Delta \mathrm{Ct}$ method for relative quantification. Specifically, following amplification, standard curves were constructed from samples used in a series of consecutive dilutions for both the gene of interest (GF) and the internal control (RPL13A). Growth factor and RPL13A amplification efficiencies were the same, reaching $100 \%$. Sample data were first normalized against variation in sample quality and quantity and then normalized values were calculated as previously described (10). A 1.5-fold increased $(\geq 1.5)$ or decreased $(\leq 0.66)$ value compared to the normal tissue (calibrator) was considered to be overexpression or downregulation, respectively. Dissociation curves of the growth factors VEGFA, TGFB1, FGF2, EGF, IGF1 and the normalizer gene RPL13A are shown in Fig. 1.

Differences in mRNA levels between pterygial and conjunctival specimens were examined (independent samples t-test). Correlations between the mRNA levels for individual growth factor and clinical information, including age, altitude of residence and the duration of pterygium presence were also examined (Pearson's bivariate correlation coefficient). Differences in mRNA levels between primary and recurrent pterygia were examined with independent samples t-test. Respective differences between pterygia of different size were examined with one-way analysis of variance (ANOVA) whereas a post-hoc analysis of differences between groups was performed with Dunnett's T3 test. A statistical analysis of findings was performed using SPSS 8.0 (SPSS, Chicago, IL). Statistical significance was set at 0.05 . 
Table I. Levels of growth factors examined in pterygial and control specimens and respective levels of statistical significance (independent samples t-test).

\begin{tabular}{lccc}
\hline Factor & Pterygia & Controls & P-value \\
\hline TGFB1 & 0.0064 & 0.0093 & 0.22 \\
VEGFA & 0.0248 & 0.0030 & 0.03 \\
FGF2 & 0.0095 & 0.0054 & 0.04 \\
EGF & 0.0017 & 0.0027 & 0.13 \\
IGF1 & 0.0027 & 0.0022 & 0.56 \\
\hline
\end{tabular}

\section{Results}

The mRNA levels for FGF2 and VEGFA were significantly higher in pterygium, compared with phenotypically normal conjunctiva. Differences in the expression of other growth factors examined between pterygium and conjunctival samples were statistically not significant. The mRNA levels of growth factors studied for pterygium and conjunctival specimens with respective levels of statistical significance are shown in Table I. The correlation between patients' age, altitude of residence or the duration of pterygium presence and the levels of growth factor mRNA was statistically not significant for the factors studied. On the other hand, FGF2 and VEGFA mRNA levels were found to be significantly different in pterygia of different sizes (ANOVA F-value $=2.96$ and $\mathrm{F}$-value $=3.24, \mathrm{p}=0.03$ and $\mathrm{p}=0.03$, respectively). mRNA levels for other growth factors examined (TGFB1, EGF and IGF1) did not differ significantly between pterygia of different sizes (ANOVA). A post-hoc analysis revealed that in large pterygia of $\geq 3 \mathrm{~mm}$ growth on the corneal surface, FGF2 and VEGFA mRNA levels were significantly higher, compared with pterygia of $\leq 3 \mathrm{~mm}$ growth on the corneal surface (Dunnett's T3 test $\mathrm{p}=0.02$ for FGF2 and VEGFA). Furthermore, recurrent pterygia displayed significantly higher mRNA levels for VEGFA, compared with primary pterygia (independent samples t-test value 6.01, p=0.02). Respective differences for the other growth factors examined including TGFB1, EGF and IGF1, as well as FGF2, were statistically not significant (independent samples t-test).

\section{Discussion}

This study evaluated the mRNA levels for 5 peptide growth factors (TGFB1, VEGFA, FGF2, EGF and IGF1) in pterygia and phenotypically normal conjunctiva by employing qRTPCR and detected significantly higher levels of expression of FGF2 and VEGFA in the former, compared with the latter. Furthermore, FGF2 and VEGFA expression was significantly higher in more advanced pterygia, compared with less advanced ones, whereas VEGFA was also significantly correlated with postoperative recurrence. Results support the involvement of FGF2 and VEGFA in pterygium pathogenesis and imply that pharmacological modification of the clinical behavior of pterygium may be possible with agents directed against specific growth factors, such as VEGFA.
The theory of benign neoplastic development, possibly originating from genetically altered limbal epithelial cells (LEC) as a mechanism for pterygium pathogenesis has gained ground (6). The predisposition to this transformation may be partly inherited, but environmental factors such as viral infections or exposure to ultraviolet (UV) radiation, also play an important role (5). Pterygium epithelial cells (PEC) and LEC, but not normal conjunctival epithelial cells (CEC), respond to UVB exposure by an enhanced production of metalloproteinase-1 (MMP-1), an enzyme involved in the turnover of extracellular matrix, supporting the concept of UV-induced genetic trauma to LEC as a pathogenetic mechanism for pterygium (11). UV-mediated genetic trauma may also affect the expression of various cytokines, growth factors and growth factor receptors (6). The presence of such factors in pterygium has been evaluated in previous studies using various methods including immunohistochemistry and ELISA in cell cultures (12). This study focused on the evaluation of five growth factors (TGFB1, VEGFA, FGF, IGF1 and EGF), which had previously been shown to be individually affected in pterygium (6), and employed qRT-PCR to detect possible alterations in the expression of these factors in pterygium, compared with normal conjunctiva as well as potential clinical associations of molecular findings. The fact that the mean altitude of residence was not significantly different between patients with pterygium and the control group, and that normal conjunctival samples were collected from the area corresponding to pterygium specimens (nasal limbus) implies that the control samples examined were exposed to the same levels of UV radiation, compared with pterygium specimens. Age and gender distribution did not differ significantly between the study and control groups, implying that the groups were epidemiologically comparable.

FGF2 is a heparin-binding growth factor (its activity is regulated by heparin and heparin sulphate) which is involved in wound healing and angiogenesis $(6,13)$. Previous studies have reported the UV-mediated induction of FGF2 in corneal epithelium in animal models (14). The significantly higher levels of FGF2 expression in pterygia compared with normal conjunctiva detected in the present study is in agreement with the previous studies and imply that FGF2 may be involved in pterygium development. FGF2 expression was significantly higher in larger pterygia of $>3 \mathrm{~mm}$ on the corneal surface, compared with pterygia of smaller size, implying that FGF2 may be involved in the clinical behavior of some pterygia and in particular with the tendency of some pterygia to display progressive growth. Altitude of residence (which is in turn associated with the levels of exposure to UV radiation) (7) did not correlate with FGF2, which may be attributed to either the relatively small number of patients studied or to the fact that these patients (irrespective of their specific location of residence) were exposed to environmental factors such as high levels of sunshine.

VEGFA is present in normal and inflamed or vascularized cornea (6). Previous studies have reported increased levels of VEGFA in skin, possibly due to an up-regulation by UV radiation, as in the case of FGF2 (15). In the case of pterygium, normal LEC, but not CEC, block the capillary tube formation induced by VEGFA (16). Therefore, LEC alterations present in pterygium may result in reduced inhibition of VEGFA 
produced by conjunctival fibroblasts, leading to increased angiogenesis which is a characteristic of pterygium (6). VEGFA expression examined with qRT-PCR in the present study was significantly higher in pterygium compared with normal conjunctiva, which is in agreement with previous immunohistochemical reports $(17,18)$. Furthermore, the significant association between postoperative recurrence and VEGFA expression detected in the present study implies that VEGFA may be involved in the clinical behavior of pterygium apart from its pathogenesis. As in the case of FGF2, the significant association between pterygium size and VEGFA expression levels implies that VEGFA may also affect the clinical aggressiveness of pterygium.

The relatively small number of patients studied is a weak point in this study. On the other hand, the group of pterygia analyzed included primary and recurrent lesions. Moreover, pterygium and control groups were derived from the same population and were comparable in terms of age and gender distribution, thereby enhancing the validity of the results. Postoperative recurrence is a significant issue in pterygium management, despite sophisticated surgery or the adjunctive use of antimetabolites, such as mitomycin C (3). The use of various anti-angiogenetic factors to treat conditions associated with pathological intraocular vascular growth such as proliferative diabetic retinopathy, exudative age-related macular degeneration or neovascular glaucoma has become common (19). Such factors interfere with VEGFA and include Pegaptanib, an oligonucleotide aptamer that binds exclusively to the 165 amino-acid isoform of VEGFA, and recombinant monoclonal antibody Bevacizumab as well as its fragment Ranibizumab, both directed against VEGFA (19). Previous studies have evaluated the potential use of Bevacizumab in pterygium management $(20,21)$. However, Bevacizumab is also associated with potential serious side effects, including significant cardiovascular toxicity (22). Findings from this study imply that modification of the clinical behavior of pterygium may be possible with Bevacizumab or other anti-VEGFA administration, potentially reducing the risk of recurrence or aggressive clinical behavior.

In conclusion, ophthalmic pterygium displays significantly higher levels of FGF2 or VEGFA expression than phenotypically normal conjunctiva. The correlation of VEGFA expression in pterygium with pterygium size and postoperative recurrence implies that anti-VEGF factors may be used in the management of selected cases of pterygium.

\section{References}

1. Hilgers J: Pterygium: its incidence, heredity and etiology. Am J Opthalmol 50: 635-644, 1960.

2. Coroneo MT: Pterygium as an indicator of ultraviolet insulation: a hypothesis. Br J Ophthalmol 77: 734-739, 1993.

3. Gans L: Surgical Treatment of Pterygium. In: Focal Points. 0 no. 12. American Academy of Ophthalmology, San Francisco, 1996.

4. Kaimbo K: Surgical treatment of pterygium: 42 cases of excision. J Fr Ophtalmol 11: 335-338, 1988.

5. Detorakis ET, Drakonaki EE and Spandidos DA: Molecular genetic alterations and viral presence in ophthalmic pterygium (Review). Int J Mol Med 6: 35-41, 2000.
6. Di Girolamo N, Chui J, Coroneo MT and Wakefield D: Pathogenesis of pterygia: role of cytokines, growth factors, and matrix metalloproteinases. Prog Retin Eye Res 23: 195-228, 2004.

7. Detorakis ET, Sourvinos G, Tsamparlakis J and Spandidos DA: Evaluation of loss of heterozygosity and microsatellite instability in human pterygium: clinical correlations. Br J Ophthalmol 82: 1324-1328, 1998.

8. Zaravinos A, Bizakis J, Soufla G, Sourvinos G and Spandidos DA: Mutations and differential expression of the ras family genes in human nasal polyposis. Int J Oncol 31: 1051-1059, 2007.

9. Soulitzis N, Karyotis I, Delakas D and Spandidos DA: Expression analysis of peptide growth factors VEGFA, FGF2, TGFB1, EGF and IGF1 in prostate cancer and benign prostatic hyperplasia. Int J Oncol 29: 305-314, 2006.

10. Zaravinos A, Soufla G, Bizakis J and Spandidos DA: Expression analysis of VEGFA, FGF2, TGFß1, EGF and IGF1 in human nasal polyposis. Oncol Rep 19: 385-391, 2008.

11. Di Girolamo N, Coroneo MT and Wakefield D: UVB-elicited induction of MMP-1 expression in human ocular surface epithelial cells is mediated through the ERK1/2 MAPKdependent pathway. Invest Ophthalmol Vis Sci 44: 4705-4714, 2003.

12. Kria L, Ohira A and Amemiya T: Growth factors in cultured pterygium fibrobroblasts: immunohistochemical and ELISA analysis. Graefes Arch Clin Exp Ophthalmol 236: 702-708, 1998.

13. Nugent MA and Iozzo RV: Fibroblast growth factor-2. Int J Biochem Cell Biol 32: 115-120, 2000.

14. Ley RD, Miska KB and Kusewitt DF: Photoreactivation of ultraviolet radiation-induced basic fibrobroblast growth factor (bFGF) and the role of bFGF in corneal lesion formation in Monodelphis domestica. Environ Mol Mutagen 38: 175-179, 2001.

15. Blaudschun R, Sunderkotter C, Brenneisen P, Hinrichs R, Peters T, Schneider L, Razi-Wolf Z, Hunzelmann N and Scharffetter-Kochanek K: Vascular endothelial growth factor causally contributes to the angiogenic response upon ultraviolet B irradiation in vivo. Br J Dermatol 146: 581-587, 2002.

16. Ma DH, Tsai RJ, Chu WK, Kao CH and Chen JK: Inhibition of vascular endothelial cell morphogenesis in cultures by limbal epithelial cells. Invest Ophthalmol Vis Sci 40: 1822-1828, 1999.

17. Lee JH, An HT, Chung JH, Kim KH, Eun HC and Cho KH: Acute effects of UVB radiation on the proliferation and differentiation of keratinocytes. Photodermatol Photoimmunol Photomed 18: 253-261, 2002.

18. Marcovich AL, Morad Y, Sandbank J, Huszar M, Rosner M, Pollack A, Herbert M and Bar-Dayan Y: Angiogenesis in pterygium: morphometric and immunohistochemical study. Curr Eye Res 25: 17-22, 2002

19. Hosseini H, Nejabat M and Khalili MR: Bevacizumab (Avastin) as a potential novel adjunct in the management of pterygia. Med Hypotheses 69: 925-927, 2007.

20. Bahar I, Kaiserman I, McAllum P, Rootman D and Slomovic A: Subconjunctival bevacizumab injection for corneal neovascularization in recurrent pterygium. Curr Eye Res 33: 23-28, 2008.

21. Daher IN and Yeh ET: Vascular complications of selected cancer therapies. Nat Clin Pract Cardiovasc Med 5: 797-805, 2008.

22. Emerson MV and Lauer AK: Emerging therapies for the treatment of neovascular age-related macular degeneration and diabetic macular edema. Bio Drugs 21: 245-257, 2007. 